\title{
Rizatriptan reduces vestibular-induced motion sickness in migraineurs
}

\author{
Joseph M. Furman • Dawn A. Marcus • \\ Carey D. Balaban
}

Received: 20 May 2010/Accepted: 12 August 2010/Published online: 23 September 2010

(C) Springer-Verlag 2010

\begin{abstract}
A previous pilot study suggested that rizatriptan reduces motion sickness induced by complex vestibular stimulation. In this double-blind, randomized, placebocontrolled study we measured motion sickness in response to a complex vestibular stimulus following pretreatment with either rizatriptan or a placebo. Subjects included 25 migraineurs with or without migraine-related dizziness (23 females) aged 21-45 years ( $31.0 \pm 7.8$ years). Motion sickness was induced by off-vertical axis rotation in darkness, which stimulates both the semicircular canals and otolith organs of the vestibular apparatus. Results indicated that of the 15 subjects who experienced vestibular-induced motion sickness when pretreated with placebo, 13 showed a decrease in motion sickness following pretreatment with rizatriptan as compared to pretreatment with placebo $(P<0.02)$. This significant effect was not seen when subjects were exposed to more provocative vestibular stimulation. We conclude that the serotonin agonist, rizatriptan, reduces vestibular-induced motion sickness by influencing serotonergic vestibular-autonomic projections.
\end{abstract}

J. M. Furman $(\bowtie) \cdot$ C. D. Balaban

Department of Otolaryngology,

Eye and Ear Institute, University of Pittsburgh

School of Medicine, 203 Lothrop Street,

Pittsburgh, PA 15213, USA

e-mail: furmanjm@upmc.edu

C. D. Balaban

e-mail: cbalaban@pitt.edu

D. A. Marcus

Department of Anesthesiology,

University of Pittsburgh School of Medicine,

Pain Medicine, Centre Commons Building,

Suite 400, 5750 Centre Avenue, Pittsburgh, PA 15206, USA
Keywords Migraine $\cdot$ Motion sickness $\cdot$ Triptans

\section{Introduction}

Motion sickness refers to a combination of autonomic and cognitive symptoms and signs induced by exposure to certain types of movement [1]. Although the definition of motion sickness has been expanded to include stimuli beyond pure vestibular stimulation, in the present study motion sickness was induced by a complex vestibular stimulation in darkness. The subjects in the present report include individuals with migraine headache. Of note is that some of the symptoms associated with migraine overlap with symptoms of motion sickness such as upper abdominal sensations, sleepiness, apathy, dizziness, cold sweating, increased salivation, pallor, and headache $[2,3]$. It is also notable that motion sickness occurs in approximately $50 \%$ of patients with migraine [4]. In this study, we only included subjects who had both migraine and motionsickness susceptibility. The pathophysiologic basis for the high incidence of motion sickness in migraine is unknown. This lack of understanding, in part, motivated the present study. Drummond [5] found that 30-40\% of persons with migraine report motion sickness with exposure to vestibular stimulation and that motion-sickness susceptibility, like migraine itself, is found more often in females [6].

Motion sickness can have a significant negative impact on an individual's quality of life especially if the motion sickness interferes with a person's ability to get to and from work, interferes with business travel, or interferes with leisure activities.

The neurophysiologic basis of motion sickness is unknown; however, several neurotransmitters seem to be important including histamine, acetylcholine, noradrenaline, 
and serotonin. The importance of these neurotransmitters for motion sickness is based on classes of medication that can be effective for treating motion sickness including antihistamines, scopolamine, amphetamines, and serotonin agonists and antagonists [7-9]. Our recent pilot studies also suggest that serotonin may be an important neurotransmitter in the neural pathways that underlie motion sickness in that the serotonin 1B/1D agonist, rizatriptan, appears to interfere with the potentiation of visually induced motion sickness by cranial pain [10].

The purpose of this study was to test the hypothesis that serotonin agonists known to benefit migraine headache also reduce vestibular-induced motion sickness. For this study, we selected subjects who had both migraine and motionsickness susceptibility. By addressing this hypothesis, we aimed to elucidate the neural mechanisms underlying motion sickness in humans. We also aimed to assess a potential remedy for motion sickness in migraineurs with high motion-sickness susceptibility.

\section{Methods}

Prior to initiating this double-blind, randomized, placebocontrolled study, the protocol and consent form were approved by the Institutional Review Board of the University of Pittsburgh. Advertisements were used to recruit healthy adult migraineurs with a history of motion-sickness susceptibility who had previously used triptans without adverse effects. An informed consent was obtained from all the subjects prior to enrollment.

\section{Subjects}

This study was designed to evaluate 25 subjects with migraine with or without an additional diagnosis of migraine-related dizziness $[11,12]$. Eligible subjects included healthy adult migraineurs aged 21-45 years who had previously used and tolerated any triptan and who had reported a history of motion sickness with either actual motion or visually induced motion perception. A boardcertified neurologist confirmed a diagnosis of migraine based on IHS criteria [13]. All enrolled subjects were also evaluated using the structured interview for migrainerelated dizziness [14, 15] and a clinical assessment based on the criteria of Neuhauser et al. [11, 12]. Subjects were excluded from the study if they were pregnant, had a history of cardiac disease, hypercholesterolemia, complicated migraine, triptan intolerance, a family history of myocardial infarction before age 45 in a first-degree relative, a weight greater than $250 \mathrm{lb}$, corrected vision worse than 20/40 OU, an active otologic disorder such as Meniere's disease, constant dizziness or vestibular symptoms, a previously identified vestibular abnormality such as benign positional vertigo or an allergy or intolerance to gelatin. Subjects using a beta-blocking agent or monamine oxidase inhibitor in the past 2 weeks or who were using other medications such as ergotamine were further excluded. Additional exclusion criteria included clinically significant abnormalities on ocular-motor and vestibulo-ocular testing, which was performed on a separate visit prior to randomization. Testing included video-oculographic recordings of saccades, pursuit, and optokinetic nystagmus, a search for positional, gaze-evoked, and spontaneous nystagmus, binaural bithermal caloric testing, and earth-vertical axis rotation. Audiometric testing was also performed. Standard techniques were used and subjects were excluded based on established normative data [16]. In order to enroll 26 subjects, 27 subjects underwent screening tests 1 of whom was unable to complete the two experimental visits described below because of elevated blood pressure while receiving placebo. The 25 subjects who completed the study consisted of 23 females and 2 males with an overall age range of 21-45 years with an average age of $31.0 \pm 7.8$ years. Of these 25 subjects, 12 subjects met the criteria for migraine-related dizziness (see Table 1).

Two experimental visits were scheduled for each subject. Each subject was randomized to receive a blinded dose of the study drug, rizatriptan $10 \mathrm{mg}$, on their first or second visit and a placebo for the other visit. The subjects, investigators, and technicians were all blinded to treatment type. The rizatriptan and placebo were blinded in identical capsules by a central pharmacy, which determined and maintained the randomization assignment and labeled each blinded medication with subject numbers. Subjects were required to be headache-free and triptan-free for at least 1 week prior to each experimental visit. After receiving the study drug or placebo, subjects were idle for $2 \mathrm{~h}$ during which their blood pressure was monitored and adverse events recorded. Then, each subject underwent a motionsickness provocation and assessment protocol.

The motion-sickness provocation and assessment protocol began with a baseline assessment of motion sickness and subjective distress. Motion sickness was judged using the motion-sickness scale developed by Graybiel [3], which rates seven subjective and objective signs and symptoms of motion sickness. Subjective units of distress were rated $0-10$ based on the method of Wolpe [17]. Subjects rated the severity of motion sickness and subjective units of distress before and after each rotation. If a subject reached a motion-sickness score of 16 or greater, the experimental visit was terminated. Following the baseline assessment, sinusoidal-earth-vertical earth axis rotation in darkness at $0.05 \mathrm{~Hz}$ was performed to assess post-drug/post-placebo vestibular-ocular reflex (VOR) 
Table 1 Subject Demographics

\begin{tabular}{|c|c|c|c|c|c|c|}
\hline Subject number & Age & Gender & Aura/no aura & Migraine-related dizziness? & Motion-sickness type & Prior triptans used \\
\hline $1 *$ & 26 & Female & Aura & Yes & Vestibular, visual & Rizatriptan, sumatriptan \\
\hline 2 & 33 & Female & Aura & No & Visual & Eletriptan \\
\hline $3 *$ & 26 & Female & Aura & Yes & Vestibular & Eletriptan, rizatriptan, sumatriptan \\
\hline 4 & 26 & Male & No aura & Yes & Vestibular, visual & Rizatriptan, sumatriptan \\
\hline $5^{*}$ & 45 & Female & No aura & No & Vestibular & Sumatriptan \\
\hline $6^{*}$ & 25 & Female & Aura & Yes & Vestibular, visual & Sumatriptan \\
\hline $7 *$ & 40 & Female & No aura & No & Vestibular & Eletriptan, rizatriptan, sumatriptan \\
\hline $8^{*}$ & 21 & Female & Aura & Yes & Visual & Sumatriptan \\
\hline 9 & 25 & Female & No aura & No & Vestibular, visual & Sumatriptan \\
\hline $10 *$ & 22 & Female & No aura & Yes & Vestibular, visual & Sumatriptan \\
\hline $11 *$ & 24 & Female & No aura & Yes & Vestibular & Sumatriptan \\
\hline $12 *$ & 28 & Female & Aura & Yes & Vestibular & Sumatriptan \\
\hline $13 *$ & 31 & Female & Aura & No & Vestibular & Naratriptan \\
\hline 14 & 30 & Female & Aura & Yes & Vestibular, visual & Sumatriptan \\
\hline 15 & 27 & Female & Aura & No & Vestibular & Rizatriptan, sumatriptan \\
\hline $16^{*}$ & 41 & Female & Aura & Yes & Vestibular, visual & Sumatriptan \\
\hline 17 & 24 & Female & Aura & No & Vestibular & Sumatriptan \\
\hline 18 & 25 & Female & Aura & Yes & Vestibular & Frovatriptan \\
\hline $19 *$ & 41 & Male & No aura & Yes & Vestibular & Sumatriptan \\
\hline 20 & 25 & Female & No aura & No & Vestibular, visual & Sumatriptan \\
\hline $21^{*}$ & 42 & Female & No aura & No & Vestibular, visual & Sumatriptan \\
\hline 22 & 26 & Female & No aura & No & Vestibular, visual & Eletriptan, sumatriptan \\
\hline $23 *$ & 40 & Female & No aura & No & Vestibular, visual & Rizatriptan, sumatriptan \\
\hline 24 & 41 & Female & Aura & No & Vestibular & Rizatriptan, sumatriptan \\
\hline $25^{*}$ & 42 & Female & Aura & No & Visual & Rizatriptan, sumatriptan \\
\hline
\end{tabular}

* Included for statistical analysis

function, i.e. VOR gain and phase. The complex vestibular stimulation to induce motion sickness consisted of offvertical axis rotation in darkness either in the clockwise or counterclockwise direction at constant velocity. By using a "rotate, then tilt" paradigm [18] the stimulus primarily activated the otolith organs of the inner ear and produced motion sickness in susceptible subjects [19]. Off-vertical axis rotation was continued for approximately $50 \mathrm{~s}$. If the subjects could tolerate additional stimulation, i.e. their motion-sickness score was $<16$, following a 2 min rest, they were then exposed to a second off-vertical axis rotation in the direction opposite to that of the first off-vertical axis rotation. This second stimulus, in general, provokes motion sickness in most subjects [18]. During both earthvertical and off-vertical axis rotations, eye movements were recorded with electro-oculography.

\section{Data analysis}

Motion-sickness scores and subjective units of distress were analyzed by computing the differences between baseline (i.e. pre-earth-vertical axis rotation) and poststimulus values. Eye-movement data were analyzed using standard techniques to compute gain and phase for earth-vertical axis rotations and modulation and bias components for off-vertical axis rotations [16]. At the completion of this analysis, the data were unblinded. It was determined that 10 of the 25 subjects developed negligible motion sickness induced by the first off-vertical axis rotation following pre-medication with placebo. The data from these subjects were not analyzed further. Statistical analysis of the change in motion-sickness scores was performed using a non-parametric paired samples sign test under the null hypothesis that the median baseline and post-stimulus scores are equal. The number of subjects displaying a subjective improvement in motion sickness was compared against the true value (i.e. 8 of 15 subjects with improvement) that would be expected if no rizatriptan treatment effect existed. A one-tailed type I error rate of 0.05 was used to judge the significance of improvement in motion-sickness scores. 


\section{Results}

All 25 subjects who completed the study could undergo at least one off-vertical rotation at each of the two visits. Three subjects had motion-sickness scores of 16 or greater following the first off-vertical axis rotation during one or both visits and thus could not undergo a second off-vertical axis rotation on that visit. For one of these subjects, this occurred only during the placebo visit; for two of these subjects, this occurred only during the rizatriptan visit. Motion-sickness scores following the first off-vertical axis rotation referenced to baseline motion-sickness score are shown in Fig. 1a. Note that the data are shown only for 15 of the 25 subjects, namely, those subjects who experienced a motion-sickness score that was more than 3 points higher than baseline following their first offvertical axis rotation on the experimental visit during which they received placebo. Note that 13 of these 15 subjects experienced decreased motion sickness with rizatriptan as compared to placebo, which is a statistically significant result ( $P<0.02$, sign test, one-tailed). Note further that the status of the patients regarding the presence or absence of a history of migraine-related dizziness did not appear to influence the effect of rizatriptan. When these same individuals were exposed to a second off-vertical axis rotation, there was no apparent benefit from rizatriptan regarding motion-sickness score referenced to baseline motion sickness (Fig. 1b). Although there was no significant effect of rizatriptan on motion-sickness score following the second off-vertical axis rotation, there was a trend for subjects without migrainerelated dizziness to show a benefit with rizatriptan as compared to placebo (4 of 5 subjects). A further analysis of motion-sickness score was performed using the ten subjects who did not achieve significant motion sickness following the first off-vertical axis rotation on the experimental visit during which they received placebo. Of these ten individuals, three individuals experienced significant motion sickness following the second off-vertical axis rotation on the experimental visit during which they received placebo. Two of these individuals experienced more motion sickness following the second offvertical axis rotation on the visit during which they received rizatriptan. An analysis of subjective units of distress indicated that there was no apparent difference between the effects of rizatriptan and placebo (See Fig. 2).

Eye-movement parameters were analyzed for evidence of a consistent effect of rizatriptan on the VOR or a correlation between motion sickness and the VOR. No consistent effects were found.

\section{Discussion}

The results of this study corroborate the implications of our prior pilot study, which suggested that rizatriptan provided
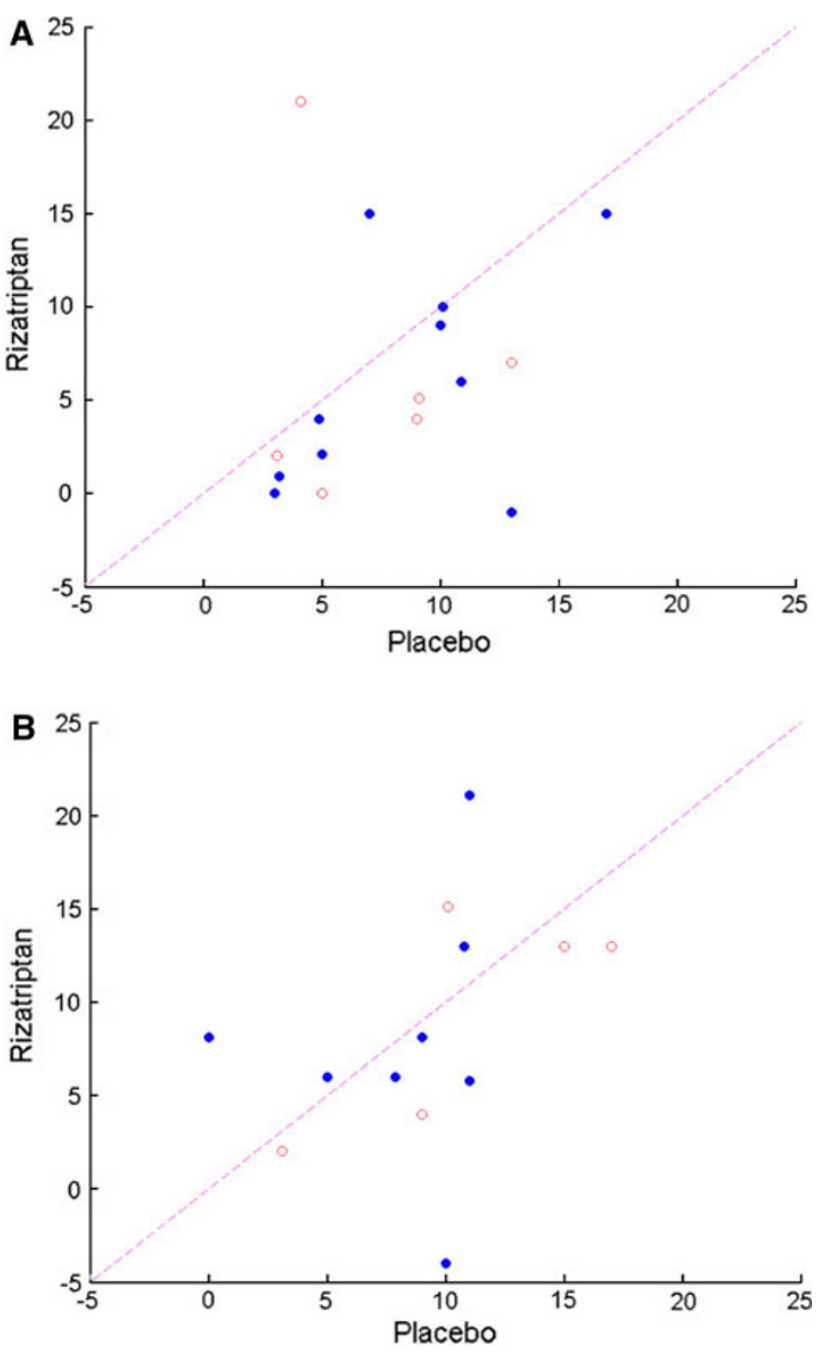

Fig. 1 a Motion-sickness score following pretreatment with rizatriptan vs. motion-sickness score following pretreatment with placebo in response to a complex vestibular stimulus, i.e. off-vertical axis rotation. Scores represent the increase in motion sickness above prestimulus baseline. Note that data are shown only from those subjects whose vestibular-induced increase in motion-sickness score exceeded 3 after pretreatment with placebo. Data from subjects with migrainerelated dizziness are shown as filled circles. Data from subjects without migraine-related dizziness are shown as open circles. Note that for 13 of 15 subjects that the increase in motion-sickness score above baseline was greater following pretreatment with placebo as compared to that seen following treatment with rizatriptan. b Motionsickness scores following a second off-vertical axis rotation. Data are shown for those subjects who could complete a second off-vertical axis rotation following pretreatment with rizatriptan and following pretreatment with placebo. Note the lack of an obvious effect of rizatriptan

protection against motion sickness induced by complex vestibular stimulation in migraineurs [20]. In the present study, which also assessed migraineurs with a history of motion sickness, an additional diagnosis of migraine-related dizziness did not affect outcome aside from a trend toward a protective effect of rizatriptan in subjects without 


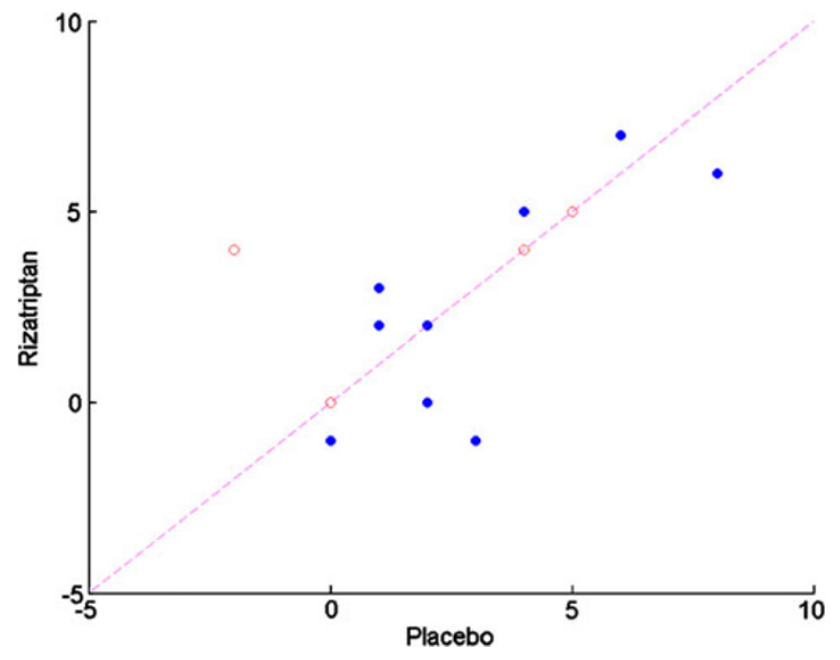

Fig. 2 Subjective units of distress after off-vertical axis rotation following pretreatment with rizatriptan vs. placebo. Note that data are shown for those subjects whose data are illustrated in Fig. 1. Note the absence of a consistent influence of rizatriptan

migraine-related dizziness following a second off-vertical axis rotation. Possibly, a protective effect was found in subjects with and without migraine-related dizziness because all subjects were individuals with motion-sickness susceptibility. The lack of an effect of pretreatment of rizatriptan on motion sickness in response to a second offvertical axis rotation suggests a type of dose-response mechanism in that rizatriptan at a single dose provided protection for a lower level of stimulation but not a higher level of motion-sickness provocation. The trend for subjects without migraine-related dizziness to gain benefit from rizatriptan despite a high level of motion-sickness provocation suggests that subjects with migraine-related dizziness may be more susceptible to motion sickness [21, 22]. Our results suggest that rizatriptan may increase the threshold for producing motion sickness. The second offvertical axis rotation may have been a suprathreshold stimulus despite rizatriptan, especially in subjects with migraine-related dizziness. This concept is consistent with the recent suggestion that motion-sickness susceptibility and migraine may be related to an innate vestibular hypersensitivity [23] and the expression of 5- $\mathrm{HT}_{1 \mathrm{~B}}$ and 5-HT receptor targets of triptans by most vestibular ganglion cells [24].

Our study also suggests that rizatriptan did not increase subjective units of discomfort. The side effects of rizatriptan can include fatigue, somnolence, head and neck pressure, nonspecific dizziness, and nausea, and thus might have been expected to increase subjective units of discomfort despite its benefit for reduction of motion sickness. Evidently, the level of distress caused by side effects of rizatriptan was offset by the reduction in motion sickness following pretreatment with rizatriptan. We speculate that the effect of rizatriptan was mediated via its effects on vestibulo-autonomic rather than vestibulo-ocular pathways.

Our prior study of rizatriptan suggested that the eyemovement response during off-vertical axis rotation was influenced by pretreatment with rizatriptan. Specifically, our study suggested that the bias component of the eyemovement response to off-vertical axis rotation was reduced following pretreatment with rizatriptan. The current study does not support this finding in a larger group of subjects. In that the bias component reflects central nervous system processing of peripheral vestibular activity, the absence of a reduction in the magnitude of the bias component suggests that rizatriptan does not interfere with the so called "velocity storage system" of central vestibuloocular processing. In addition, there were no consistent changes in VOR gain and phase following pretreatment with rizatriptan. This further suggests that the effect of rizatriptan on motion sickness is not mediated via changes in "velocity storage."

The present study has implications for understanding the pathophysiology of migraine-related dizziness and motion sickness in migraineurs. Recent studies indicate a remarkable degree of neurochemical similarity between nociceptive trigeminal ganglion cells and vestibular ganglion cells. For example, most vestibular ganglion cells express functional biomarkers associated with nociception, such as TRPV1 receptors [25], substance P [26], P2X purinergic receptors [27], and both the 5- $\mathrm{HT}_{1 \mathrm{~B}}$ and $5-\mathrm{HT}_{1 \mathrm{D}}$ receptors [24]. Moreover, plasma extravasation occurs in the vestibular periphery at the same time as extravasation in the meninges in a murine model of neurogenic migraine [28]. Finally, functional imaging studies report that increased cerebral blood flow in a region of the dorsal and dorsolateral pons accompanies both spontaneous and glyceryl trinitrate induced migraine attacks [29-33]; the implicated region appears to include portions of the vestibular nuclei, medial parabrachial nucleus, locus coeruleus and dorsal raphe nuclei.

There is also a considerable overlap of symptoms between motion sickness and migraine. Motion sickness describes a progression of symptoms evoked by real or virtual motion. It begins with the 'sopite syndrome' [34], a form of motion sickness that is characterized by yawning, drowsiness, disinclination for either physical or mental work, and lack of participation in group activities. The symptoms can progress to vague abdominal discomfort, hyperventilation, salivation, pallor, profuse cold sweating, somnolence, and nausea. Vomiting and retching can also ensue. There is a striking overlap with symptoms of migraine and passive emotional coping responses to pain $[35,36]$. Hence, the efficacy of rizatriptan against motion sickness is consistent with the hypothesis that engagement of migraine-related mechanisms, such as trigemino-vascular reflexes and central pain 


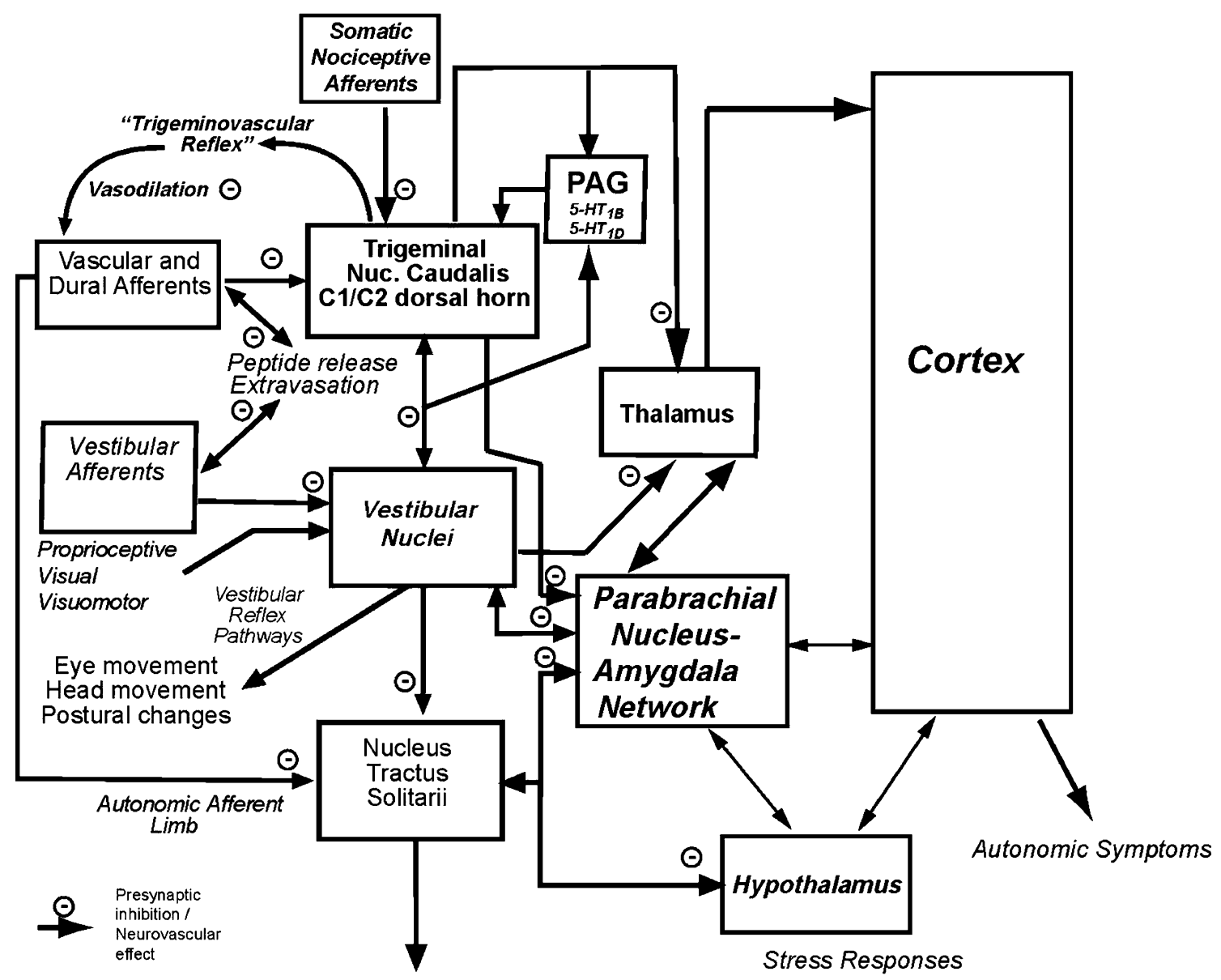

Fig. 3 Schematic diagram to explain effects of rizatriptan on motion sickness in migraineurs. The enhanced motion-sickness susceptibility in migraineurs is hypothesized to reflect the net vestibular nuclear and solitary nucleus effects of vestibular stimulation. The trigeminal nociceptive pathway and vestibular nuclei also contribute ascending thalamic connections, which contribute to autonomic symptoms via projections to the cerebral cortex. Spinal trigeminal nuclear and vestibular nuclear neurons also project to the parabrachial nucleus. Stress responses and vasopressin release are mediated by interconnections of the parabrachial pathways with the hypothalamus. We

pathways, may contribute to the generation of motion sickness.

As summarized in Fig. 3, the enhanced motion-sickness susceptibility in migraineurs may be a function of the net vestibular nuclear and solitary nucleus effects of vestibular stimulation. Triptans have been shown to reduce nociceptive activation in animal studies through actions on somatic, vascular and dural afferents, and central trigeminal and solitary nucleus neurons [37-40]. These actions appear to be primarily by $5-\mathrm{HT}_{1 \mathrm{~B}}$ and $5-\mathrm{HT}_{1 \mathrm{D}}$-mediated presynaptic inhibition [40, 41]. Activation of 5-HT $1 \mathrm{~B}$ and $5-\mathrm{HT}_{1 \mathrm{D}}$ receptors in the ventrolateral periaqueductal gray inhibit trigeminal and solitary nucleus nociceptive responses [42] and affect passive coping responses to pain [36] that resemble the prodromal features of motion sickness. We propose that motion-sickness sensitivity is propose that motion-sickness sensitivity is decreased by the actions of rizatriptan on $5-\mathrm{HT}_{1 \mathrm{~B}}$ and $5-\mathrm{HT}_{1 \mathrm{D}}$ receptors on (1) vasculature (e.g. blocking vasodilatation), (2) vestibular ganglion cells, (3) trigeminal ganglion cells (for somatic, vascular and dural afferents), (4) several brainstem pathways and projections, and (5) neurons in the periaqueductal gray $(P A G)$. The primary effect of rizatriptan is via presynaptic inhibition of terminals that can use either excitatory amino acids or GABA. These presynaptic effects can occur at primary afferent and in CNS pathways

decreased by the additional actions of rizatriptan on $5-\mathrm{HT}_{1 \mathrm{~B}}$ and $5-\mathrm{HT}_{1 \mathrm{D}}$ receptors on vestibular ganglion cells [24] and vestibular nuclear neurons [43], which would be expected to raise the threshold for eliciting symptoms. Rizatriptan can also have direct vascular effects via $5-\mathrm{HT}_{1 \mathrm{~B}}$ and 5-HT $1 \mathrm{D}$ receptors, attenuating vasodilatation and extravasation from cerebral and inner ear vasculature. Thus, these findings are consistent with the concept that parallel activation of vestibular and cranial nociceptive pathways may contribute to the otologic features of migraine-related dizziness. More generally, they provide a basis for an emerging view that motion sickness may be a form of interoceptive pain.

At present, there are several symptomatic treatments for motion sickness including vestibular suppressant medications such as meclizine. Individuals especially prone to 
motion sickness often avoid provocative activities. Preventive treatments include scopolamine, promethazine, and antihistamines. These medications usually cause sedation or other unwanted effects.

The reduction in motion sickness following pretreatment with rizatriptan suggests that rizatriptan may provide benefit for migraineurs who suffer from motion sickness. As noted above, the mechanism whereby rizatriptan reduces motion sickness may be related to an alteration in the threshold for motion sickness based on serotonergic mechanisms. Of interest is that no changes in the VOR were found in response to rizatriptan despite effects on motion sickness. This finding does not support the current theories of motion sickness proffered by Dai et al. [44] and studied by Jeong et al. [23] that so called "velocity storage" in the VOR is fundamental to motion sickness. Possibly, migraineurs who cannot avoid motion sickness provoking environments could pre-medicate with rizatriptan with benefit. Further research would be required to establish this potential benefit in that the present study is laboratory-based and may not entirely reflect the level of symptoms produced in a natural environment.

Acknowledgments The authors thank Anita Lieb and Susan Strelinski for technical assistance and Dr. Gregory Marchetti for statistical analysis. This work was supported by an investigatorinitiated award from Merck \& Co., Inc.

Conflict of interest None.

\section{References}

1. Irwin JA (1881) The pathology of sea-sickness. Lancet 2:907-909

2. Marcus DA, Furman JM, Balaban CD (2005) Motion sickness in migraine sufferers. Expert Opin Pharmacother 6(15):2691-2697

3. Graybiel A, Wood CD, Miller EF, Cramer DB (1968) Diagnostic criteria for grading the severity of acute motion sickness. Aerosp Med 39(5):453-455

4. Kayan A, Hood JD (1984) Neuro-otological manifestations of migraine. Brain 107(Pt 4):1123-1142

5. Drummond PD (2005) Triggers of motion sickness in migraine sufferers. Headache 45(6):653-656

6. Turner M, Griffin MJ, Holland I (2000) Airsickness and aircraft motion during short-haul flights. Aviat Space Environ Med 71(12):1181-1189

7. Yates BJ, Miller AD, Lucot JB (1998) Physiological basis and pharmacology of motion sickness: an update. Brain Res Bull 47(5):395-406

8. Takeda N, Morita M, Horii A, Nishiike S, Kitahara T, Uno A (2001) Neural mechanisms of motion sickness. J Med Invest 48(1-2):44-59

9. Javid FA, Naylor RJ (2002) The effect of serotonin and serotonin receptor antagonists on motion sickness in Suncus murinus. Pharmacol Biochem Behav 73(4):979-989
10. Furman JM, Marcus DA (2009) A pilot study of rizatriptan and visually-induced motion sickness in migraineurs. Int $\mathrm{J}$ Med Sci 6(4):212-217

11. Neuhauser H, Leopold M, von Brevern M, Arnold G, Lempert T (2001) The interrelations of migraine, vertigo, and migrainous vertigo. Neurology 56(4):436-441

12. Abu-Arafeh I, Russell G. Paroxysmal vertigo as a migraine equivalent in children: a population-based study. Cephalalgia 15(1):22-5 (discussion 4)

13. The International Classification of Headache Disorders (2004) 2nd edn. Cephalalgia 24(Suppl 1):9-160

14. Marcus DA, Kapelewski C, Rudy TE, Jacob JG, Furman JM (2004) Diagnosis of migrainous vertigo: validity of a structured interview. Med Sci Monit 10(5):CR197-201

15. Marcus DA, Kapelewski C, Jacob RG, Rudy TE, Furman JM (2004) Validation of a brief nurse-administered migraine assessment tool. Headache 44(4):328-332

16. Furman JM, Wuyts F (2003) Vestibular test techniques. In: Luxon LM, Martini A, Furman JM, Stephens D (eds) Textbook of audiological medicine. Martin Dunitz, London, pp 735-746

17. Wolpe J (1982) The practice of behavior therapy. Pergamon Press, New York

18. Furman JM, Schor RH, Schumann TL (1992) Off-vertical axis rotation: a test of the otolith-ocular reflex. Ann Otol Rhinol Laryngol 101(8):643-650

19. Denise P, Etard O, Zupan L, Darlot C (1996) Motion sickness during off-vertical axis rotation: prediction by a model of sensory interactions and correlation with other forms of motion sickness. Neurosci Lett 203(3):183-186

20. Marcus DA, Furman JM (2006) Prevention of motion sickness with rizatriptan: a double-blind, placebo-controlled pilot study. Med Sci Monit 12(1):PI1-7

21. Evans RW, Marcus D, Furman JM (2007) Motion sickness and migraine. Headache 47(4):607-610

22. Neuhauser HK, Radtke A, von Brevern M, Feldmann M, Lezius F, Ziese T et al (2006) Migrainous vertigo: prevalence and impact on quality of life. Neurology 67(6):1028-1033

23. Jeong SH, Oh SY, Kim HJ, Koo JW, Kim JS (2009) Vestibular dysfunction in migraine: effects of associated vertigo and motion sickness. J Neurol 257(6):905-912

24. Ahn S-K, Balaban CD (2010) Distribution of 5-HT1B and 5HT1D receptors in the inner ear. Brain Res 1346:92-101

25. Kitahara T, Li HS, Balaban CD (2005) Changes in transient receptor potential cation channel superfamily $\mathrm{V}$ (TRPV) mRNA expression in the mouse inner ear ganglia after kanamycin challenge. Hear Res 201(1-2):132-144

26. Kevetter GA, Leonard RB (2002) Molecular probes of the vestibular nerve. II. Characterization of neurons in Scarpa's ganglion to determine separate populations within the nerve. Brain Res 928(1-2):18-29

27. Xiang Z, Bo X, Burnstock G (1999) P2X receptor immunoreactivity in the rat cochlea, vestibular ganglion and cochlear nucleus. Hear Res 128(1-2):190-196

28. Koo JW, Balaban CD (2006) Serotonin-induced plasma extravasation in the murine inner ear: possible mechanism of migraineassociated inner ear dysfunction. Cephalalgia 26(11):1310-1319

29. Afridi SK, Giffin NJ, Kaube H, Friston KJ, Ward NS, Frackowiak RS et al (2005) A positron emission tomographic study in spontaneous migraine. Arch Neurol 62(8):1270-1275

30. Afridi SK, Matharu MS, Lee L, Kaube H, Friston KJ, Frackowiak RS et al (2005) A PET study exploring the laterality of brainstem activation in migraine using glyceryl trinitrate. Brain $128(\mathrm{Pt}$ 4):932-939

31. Afridi SK, Goadsby PJ (2006) Neuroimaging of migraine. Curr Pain Headache Rep 10(3):221-224 
32. May A, Matharu M (2007) New insights into migraine: application of functional and structural imaging. Curr Opin Neurol 20(3):306-309

33. May A (2006) A review of diagnostic and functional imaging in headache. J Headache Pain 7(4):174-184

34. Graybiel A, Knepton J (1976) Sopite syndrome: a sometimes sole manifestation of motion sickness. Aviat Space Environ Med 47(8):873-882

35. Bernard JF, Bandler R (1998) Parallel circuits for emotional coping behaviour: new pieces in the puzzle. J Comp Neurol 401(4):429-436

36. Bandler R, Price JL, Keay KA (2000) Brain mediation of active and passive emotional coping. Prog Brain Res (122):331-349

37. Donaldson C, Boers PM, Hoskin KL, Zagami AS, Lambert GA (2002) The role of 5-HT1B and 5-HT1D receptors in the selective inhibitory effect of naratriptan on trigeminovascular neurons. Neuropharmacology 42(3):374-385

38. Goadsby PJ, Classey JD (2003) Evidence for serotonin (5-HT)1B, 5-HT1D and 5-HT1F receptor inhibitory effects on trigeminal neurons with craniovascular input. Neuroscience 122(2):491-498
39. Jeggo RD, Wang Y, Jordan D, Ramage AG (2007) Activation of 5-HT1B and 5-HT1D receptors in the rat nucleus tractus solitarius: opposing action on neurones that receive an excitatory vagal C-fibre afferent input. Br J Pharmacol 150(8):987-995

40. Jennings EA, Ryan RM, Christie MJ (2004) Effects of sumatriptan on rat medullary dorsal horn neurons. Pain 111:30-37

41. Matsuoka T, Hasuo H, Akasu T (2004) 5-Hydroxytryptamine 1B receptors mediate presynaptic inhibition of monosynaptic IPSC in the rat dorsolateral septal nucleus. Neurosci Res 48(3):229-238

42. Bartsch T, Knight YE, Goadsby PJ (2004) Activation of 5 -HT(1B/1D) receptor in the periaqueductal gray inhibits nociception. Ann Neurol 56(3):371-381

43. Ahn S-K, Balaban CD (2007) Regional distribution of 5-HT1A, $1 \mathrm{~B}$, and $1 \mathrm{D}$ receptors in rat vestibular nuclei $(\mathrm{VN})$. Soc Neurosci Abstr 37(\#180.5)

44. Dai M, Raphan T, Cohen B (2007) Labyrinthine lesions and motion sickness susceptibility. Exp Brain Res 178(4):477-487 